\title{
An Improved Group Sampling Plan Based on Time-Truncated Life Tests
}

\author{
Muhammad Aslam ${ }^{1, a}$, Muhammad Khalid Pervaiz ${ }^{b}$, Chi-Hyuck Jun ${ }^{c}$ \\ ${ }^{a}$ Department of Statistics, Forman Christian College University Lahore \\ ${ }^{b}$ Department of Statistics, GC University \\ ${ }^{c}$ Department of Industrial and Management Engineering, POSTECH
}

\begin{abstract}
In this paper, a new group sampling plan for the lot acceptance is proposed for the time truncated life test, which can be utilized when multi-item testers are implemented. The design parameters are found using the two-point approach such that the producer's and consumer's risks are satisfied simultaneously at the acceptable reliability level and the lot tolerance reliability level, respectively. The case of Weibull distribution is described to illustrate the procedure that can be used when the quality level is expressed by a multiple of the specified life. The advantage of the proposed plan is demonstrated by comparing with the existing plan in terms of the sample size required. The tables are constructed and some examples are given to illustrate the procedure developed here.
\end{abstract}

Keywords: Acceptance sampling, producer's risk, consumer's risk, Weibull distribution.

\section{Introduction}

Acceptance sampling plan is an inspecting procedure in statistical quality control and is used to make a decision on the submitted lot through random samples. Variables or attributes single sampling plans are widely used for these purposes. Variables sampling plans are in general more informative but they are quite limited in use to particular life distributions. On the other hand, attributes sampling plans can be developed for various distributions.

Attributes single sampling plans have been proposed for a variety of life distributions by many authors. See for an example, Goode and Kao (1961) for Weibull distribution, Gupta and Groll (1961) for gamma distributions, Gupta (1962) for normal and log-normal distributions, Tsai and Wu (2006) for a generalized Rayleigh distribution, Kantam et al. (2001) for the log-logistic distribution, and Balakrishnan et al. (2007) for a generalized Birnbaum-Saunders distribution.

In a single sampling plan items are tested one by one, but testers are available in practice, which are used to test several items simultaneously. The single and double group sampling plans for the sudden life testing are introduced by Jun et al. (2006) for the Weibull distribution. Recently, the group sampling plan for the truncated life test is proposed by Aslam and Jun (2009a) for the inverse Rayleigh and log-logistic distributions. Aslam and Jun (2009b) proposed the group sampling plan for the Weibull distribution by considering the producer's and consumer's risks at the same time. The group sampling plan is considered as more efficient than the single sampling plan in terms of cost and time to reach the final decision about the submitted lot.

The purpose of this paper is to propose an improved group sampling plan in terms of the sample size. We use the two-point approach when designing the proposed plan. Two cases are considered,

\footnotetext{
${ }^{1}$ Corresponding author: Department of Statistics, Forman Christian College University Lahore, Pakistan.

E-mail: aslam_ravian@hotmail.com
} 
one of which is the case where the acceptable reliability level and the lot tolerance reliability level are expressed by the unreliability and the other is the case where the quality levels are expressed by the mean ratio to the specified life under Weibull distribution. The rest of the paper is organized as follows: The proposed group sampling plan is given in Section 2. The designing of the proposed plan is described and tables are constructed in Section 3. In Section 4, some concluding remarks are given.

\section{An Improved Group Sampling Plan}

The acceptance criterion in the existing group sampling plan as in Aslam and Jun (2009a) requires that the number of failures should be smaller than or equal to a specified number ( $c$, say) from each group. We would like to relax the requirement to allow more failures in some groups. The test procedure under the proposed group sampling plan having the group size of $r$ is as follows:

(1) Select the number of groups $g$ and allocate predefined $r$ items to each group so that the sample size for a lot will be $n=g r$.

(2) Select the acceptance number $c(c \leq r)$ for a group and the experiment time $t_{0}$.

(3) Perform the experiment for the $g$ groups simultaneously and record the number of failures for each group.

(4) Accept the lot if the number of failures is smaller than or equal to $c$ from at least $k$ groups $(k \leq g)$. Otherwise, truncate the experiment and reject the lot.

The proposed plan has three design parameters $g, k$ and $c$, when the experiment time $t_{0}$ is fixed. If $k=g$, the proposed plan reduces to the one in Aslam and Jun (2009a, 2009b).

The lot acceptance probability under the above group sampling plan is given by

$$
L(p)=\sum_{j=k}^{g}\left(\begin{array}{l}
g \\
j
\end{array}\right) Q^{j}(1-Q)^{g-j},
$$

where $Q$ is the probability that $c$ or smaller failures are observed in a group:

$$
Q=\left[\sum_{i=0}^{c}\left(\begin{array}{l}
r \\
i
\end{array}\right) p^{i}(1-p)^{r-i}\right] .
$$

Here, $p$ is the probability that an item fails by time $t_{0}$, which is given by

$$
p=F\left(t_{0}\right)
$$

when $F$ is the cumulative distribution function of the life of an item.

\section{Design of the Proposed Plan}

The usual approach to designing the plan parameters is two-point method, which utilizes the two points on the operating characteristic(OC) curve. As in Fertig and Mann (1980), the probability of acceptance should be greater than $1-\alpha$ ( $\alpha$ is called producer's risk) at the acceptable reliability level(ARL), $p_{0}$ say, and the probability of acceptance should be smaller than $\beta$ (this is called consumer's risk) at the lot tolerance reliability level(LTRL), $p_{1}$ say. The quality level of a lot can be 
Table 1: Proposed group sampling plan indexed by ARL and LTRL $(\alpha=0.05, \beta=0.1)$

\begin{tabular}{|c|c|c|c|c|c|c|c|c|c|c|c|}
\hline \multirow{2}{*}{$p_{0}$} & \multirow{2}{*}{$p_{1}$} & \multicolumn{5}{|c|}{$r=5$} & \multicolumn{5}{|c|}{$r=10$} \\
\hline & & $c$ & $k$ & $g$ & sample size & $L\left(p_{0}\right)$ & $c$ & $k$ & $g$ & sample size & $L\left(p_{0}\right)$ \\
\hline \multirow{5}{*}{0.001} & 0.005 & 1 & 15719 & 15720 & 78600 & 0.9889 & 1 & 3549 & 3550 & 35500 & 0.9886 \\
\hline & 0.010 & 1 & 3966 & 3967 & 19835 & 0.9992 & 1 & 910 & 911 & 9110 & 0.9992 \\
\hline & 0.015 & 0 & 51 & 52 & 260 & 0.9720 & 0 & 26 & 27 & 270 & 0.9705 \\
\hline & 0.020 & 0 & 39 & 40 & 200 & 0.9829 & 0 & 19 & 20 & 200 & 0.9833 \\
\hline & 0.030 & 0 & 26 & 27 & 135 & 0.9920 & 0 & 13 & 14 & 140 & 0.9917 \\
\hline \multirow{4}{*}{0.005} & 0.025 & 1 & 652 & 653 & 3265 & 0.9883 & 1 & 156 & 157 & 1570 & 0.9869 \\
\hline & 0.050 & 1 & 101 & 101 & 505 & 0.9753 & 1 & 26 & 26 & 260 & 0.9719 \\
\hline & 0.100 & 0 & 7 & 8 & 40 & 0.9845 & 0 & 4 & 5 & 50 & 0.9784 \\
\hline & 0.150 & 0 & 5 & 6 & 30 & 0.9914 & 0 & 3 & 4 & 40 & 0.9866 \\
\hline \multirow{4}{*}{0.010} & 0.050 & 1 & 170 & 171 & 855 & 0.9875 & 1 & 43 & 44 & 440 & 0.9847 \\
\hline & 0.100 & 1 & 28 & 28 & 140 & 0.9729 & 1 & 8 & 8 & 80 & 0.9664 \\
\hline & 0.200 & 0 & 4 & 5 & 25 & 0.9782 & 0 & 2 & 3 & 30 & 0.9743 \\
\hline & 0.300 & 0 & 2 & 3 & 15 & 0.9930 & 0 & 1 & 2 & 20 & 0.9909 \\
\hline \multirow{2}{*}{0.100} & 0.250 & 1 & 9 & 10 & 50 & 0.9796 & 1 & 3 & 4 & 40 & 0.9604 \\
\hline & 0.500 & 1 & 2 & 2 & 10 & 0.9553 & 2 & 1 & 1 & 10 & 0.9885 \\
\hline
\end{tabular}

represented by the true mean if the underlying life distribution is specified. In this case, the ARL or the LTRL is expressed by a multiple of the specified life. In Section 3.1 we will determine the plan parameters when the ARL and the LTRL are specified directly in terms of unreliability. In Section 3.2, Weibull distribution is considered and the proposed plan parameters will be determined when the ARL and the LTRL are specified as multiples of the specified life.

\subsection{Design parameters indexed by $A R L$ and $L T R L$ as unreliability}

Although the unreliability is obtained by the underlying life distribution, the plan parameters can be determined independently of the underlying life distribution if the ARL $p_{0}$ and the LTRL $p_{1}$ are directly specified as unreliability. According to the statement for the two-point approach, the plan parameters $(g, k, c)$ should satisfy the following two inequalities:

$$
\begin{aligned}
& L\left(p_{0}\right)=\sum_{j=k}^{g}\left(\begin{array}{l}
g \\
j
\end{array}\right) Q_{0}^{j}\left(1-Q_{0}\right)^{g-j} \geq 1-\alpha, \\
& L\left(p_{1}\right)=\sum_{j=k}^{g}\left(\begin{array}{l}
g \\
j
\end{array}\right) Q_{1}^{j}\left(1-Q_{1}\right)^{g-j} \leq \beta,
\end{aligned}
$$

where $Q_{0}$ and $Q_{1}$ are the values of $Q$ in (2.2) corresponding to the specified ARL $p_{0}$ and LTRL $p_{1}$, respectively.

Table 1 shows the design parameters when the different combinations of ARL and LRL are specified. Two cases of the group size are considered ( $r=5$ and $r=10)$. It is assumed that the consumer's risk is 10 percent and the producer's risk is 5 percent. In this table, lot acceptance probability $L\left(p_{0}\right)$ and the sample size required were also reported.

It is observed that the number of groups and the sample size decrease rapidly as the LTRL increases. It is interesting to see that $k$ was determined by $g-1$ or $\mathrm{g}$ for all cases considered. When comparing the case of $r=5$ and the case of $r=10$, the case of $r=5$ leads to larger sample size if the ratio of $p_{1} / p_{0}$ is relatively small but to smaller sample size if the ratio is relatively large. So, there may exist another decision problem when there are alternatives in group size.

Example 1. Suppose that a manufacturer wants to adopt the proposed group sampling plan when 
Table 2: Comparison of sample sizes between two group sampling plans

\begin{tabular}{|c|c|c|c|c|c|c|}
\hline \multirow{2}{*}{$p_{0}$} & \multirow{2}{*}{$p_{1}$} & \multicolumn{2}{|c|}{$r=5$} & \multicolumn{2}{|c|}{$r=10$} & \multirow{2}{*}{$\begin{array}{c}r=1 \\
\text { Ordinary Plan }\end{array}$} \\
\hline & & Proposed Plan & Existing Plan $(k=g)$ & Proposed Plan & Existing Plan $(k=g)$ & \\
\hline \multirow{3}{*}{0.001} & 0.015 & 260 & 5270 & 270 & 2460 & 258 \\
\hline & 0.020 & 200 & 2990 & 200 & 1420 & 194 \\
\hline & 0.030 & 135 & 1355 & 140 & 660 & 129 \\
\hline \multirow{3}{*}{0.050} & 0.050 & 505 & 505 & 260 & 260 & 105 \\
\hline & 0.100 & 40 & 140 & 50 & 80 & 38 \\
\hline & 0.150 & 30 & 65 & 40 & 40 & 25 \\
\hline \multirow{3}{*}{0.010} & 0.100 & 140 & 140 & 80 & 80 & 52 \\
\hline & 0.200 & 25 & 40 & 30 & 30 & 18 \\
\hline & 0.300 & 15 & 20 & 20 & 20 & 12 \\
\hline \multirow{2}{*}{0.050} & 0.250 & 50 & 110 & 40 & 40 & 25 \\
\hline & 0.500 & 10 & 10 & 10 & 10 & 9 \\
\hline 0.100 & 0.500 & 15 & 20 & 20 & 20 & 12 \\
\hline
\end{tabular}

making a decision of accepting or rejecting the submitted lots of his products. Multi-item testers with group size of 5 are used for the test. They would like to keep the consumer's risk below 10 percent if the unreliability is 0.1 , whereas the producer's risk should be less than 5 percent when the unreliability is as low as 0.005. It is seen from Table 1 that $(g, k, c)=(8,7,0)$ for the proposed group sampling plan, which is implemented as follows: Take a sample of 40 items from a lot and allocate 5 items to 8 groups. Accept the lot if there are no failures in 7 out of 8 groups and reject, otherwise.

In order to demonstrate the advantage of the proposed group sampling plan over the existing group sampling plan, the sample size to be required can be compared. Table 2 compares the sample size for the proposed plan with the existing group sampling plan in Aslam and Jun (2009b).

It can be seen from Table 2, the proposed plan needs smaller number of sample size than in the existing plan for same values of ARL and LTRL. Particularly for $p_{0}=0.001$, the reduction in the sample size by the proposed plan is quite tremendous. The sample size for the ordinary acceptance sampling plan (the case of $r=1$ ) is also placed in Table 2 at the same values of $p_{0}$ and $p_{1}$ for the reference. However, it should not be compared with the proposed group sampling plan in terms of the sample size because the group $\operatorname{size}(r)$ is not a decision variable

\subsection{Design parameters for a Weibull distribution}

Sometimes, it may be more convenient to express the quality level in terms of the true mean rather than the reliability. This is the case particularly when the true life is to be compared with the specified life. For this purpose, however, the underlying life distribution should be known. This section illustrates how the proposed group sampling plan can be designed for the Weibull distribution when the ARL and the LTRL are expressed as multiples of the specified life.

Suppose that the lifetime of a product follows the Weibull distribution with known shape parameter. The cumulative distribution function of Weibull distribution with shape parameter $m$ and scale parameter $\lambda$ is given by

$$
F(t)=1-\exp \left(-\left(\frac{t}{\lambda}\right)^{m}\right), \quad t \geq 0 .
$$

The mean life of the Weibull distributed items is given as

$$
\mu=\left(\frac{\lambda}{m}\right) \Gamma\left(\frac{1}{m}\right)
$$


Table 3: Proposed plan for Weibull distribution with $m=2$ when $r=5$

\begin{tabular}{|c|c|c|c|c|c|c|c|c|c|c|c|}
\hline \multirow{2}{*}{$\beta$} & \multirow{2}{*}{$\begin{array}{l}\mu / \mu_{0} \\
=r_{2}\end{array}$} & \multicolumn{5}{|c|}{$a=0.5$} & \multicolumn{5}{|c|}{$a=1.0$} \\
\hline & & $c$ & $k$ & $g$ & sample size & $L\left(p_{0}\right)$ & $c$ & $k$ & $g$ & sample size & $L\left(p_{0}\right)$ \\
\hline \multirow{4}{*}{0.25} & 2 & 1 & 11 & 12 & 60 & 0.9751 & 2 & 3 & 4 & 20 & 0.9897 \\
\hline & 4 & 0 & 3 & 4 & 20 & 0.9804 & 1 & 1 & 1 & 5 & 0.9772 \\
\hline & 6 & $\uparrow$ & $\uparrow$ & $\uparrow$ & $\uparrow$ & 0.9958 & $\uparrow$ & $\uparrow$ & $\uparrow$ & $\uparrow$ & 0.9955 \\
\hline & 8 & $\uparrow$ & $\uparrow$ & $\uparrow$ & $\uparrow$ & 0.9986 & $\uparrow$ & $\uparrow$ & $\uparrow$ & $\uparrow$ & 0.9985 \\
\hline \multirow{4}{*}{0.10} & 2 & 1 & 16 & 17 & 85 & 0.9520 & 2 & 5 & 6 & 30 & 0.9757 \\
\hline & 4 & 0 & 4 & 5 & 25 & 0.9686 & 0 & 1 & 2 & 10 & 0.9526 \\
\hline & 6 & $\uparrow$ & $\uparrow$ & $\uparrow$ & $\uparrow$ & 0.9931 & $\uparrow$ & $\uparrow$ & $\uparrow$ & $\uparrow$ & 0.9893 \\
\hline & 8 & $\uparrow$ & $\uparrow$ & $\uparrow$ & $\uparrow$ & 0.9978 & $\uparrow$ & $\uparrow$ & $\uparrow$ & $\uparrow$ & 0.9965 \\
\hline \multirow{4}{*}{0.05} & 2 & 2 & 109 & 110 & 550 & 0.9942 & 2 & 5 & 6 & 30 & 0.9757 \\
\hline & 4 & 0 & 5 & 6 & 30 & 0.9548 & 0 & 1 & 2 & 10 & 0.9526 \\
\hline & 6 & $\uparrow$ & $\uparrow$ & $\uparrow$ & $\uparrow$ & 0.9899 & $\uparrow$ & $\uparrow$ & $\uparrow$ & $\uparrow$ & 0.9893 \\
\hline & 8 & $\uparrow$ & $\uparrow$ & $\uparrow$ & $\uparrow$ & 0.9967 & $\uparrow$ & $\uparrow$ & $\uparrow$ & $\uparrow$ & 0.9965 \\
\hline \multirow{4}{*}{0.01} & 2 & 2 & 152 & 153 & 765 & 0.9890 & 3 & 23 & 24 & 120 & 0.9951 \\
\hline & 4 & 1 & 19 & 19 & 95 & 0.9728 & 1 & 3 & 4 & 20 & 0.9975 \\
\hline & 6 & 0 & 7 & 8 & 40 & 0.9818 & 0 & 2 & 3 & 15 & 0.9702 \\
\hline & 8 & $\uparrow$ & $\uparrow$ & $\uparrow$ & $\uparrow$ & 0.9938 & $\uparrow$ & $\uparrow$ & $\uparrow$ & $\uparrow$ & 0.9898 \\
\hline
\end{tabular}

Note) The upward arrow $(\uparrow)$ indicates that the same value of the parameter as in the upward cell applies to the corresponding cell.

Then, the unreliability at time $t_{0}$ can be obtained from (2.3) by

$$
p=1-\exp \left(-b^{m}\left(\frac{t_{0}}{\mu}\right)^{m}\right),
$$

where

$$
b=\frac{\Gamma(1 / m)}{m} .
$$

Suppose that there exists the specified life $\mu_{0}$ of interest, beyond which the quality is desirable. Now, The quality level of a product can be expressed in terms of the mean ratio, $\mu / \mu_{0}$. It would be convenient to specify the termination time $t_{0}$ as a multiple of the specified life $\mu_{0}$. That is, we will consider $t_{0}=a \mu_{0}$ for a constant $a$. Then, the unreliability in (3.4) reduces to

$$
p=1-\exp \left(-(a b)^{m}\left(\frac{\mu}{\mu_{0}}\right)^{-m}\right) .
$$

If $r_{0}$ is the ARL as mean ratio at the producer's risk and $r_{1}$ is the LTRL at the consumer's risk, then design parameters should be obtained by satisfying the following two inequalities:

$$
\begin{aligned}
& L\left(p_{0} \mid \frac{\mu}{\mu_{0}}=r_{0}\right) \geq 1-\alpha, \\
& L\left(p_{1} \mid \frac{\mu}{\mu_{0}}=r_{1}\right) \leq \beta .
\end{aligned}
$$

We will consider $r_{1}=1$ because the acceptance of a lot should indicate the true mean life greater than the specified life at the risk of $\beta$. We will consider various ratios for $r_{0}$.

Table 3 shows the design parameter for the proposed group sampling plan with $r=5$ under the Weibull distribution having the shape parameter of 2 when the producer's risk is 5 percent. Two test 
Table 4: Proposed plan for Weibull distribution with $m=2$ when $r=10$

\begin{tabular}{|c|c|c|c|c|c|c|c|c|c|c|c|}
\hline \multirow{2}{*}{$\beta$} & \multirow{2}{*}{$\begin{array}{l}\mu / \mu_{0} \\
=r_{2}\end{array}$} & \multicolumn{5}{|c|}{$a=0.5$} & \multicolumn{5}{|c|}{$a=1.0$} \\
\hline & & $c$ & $k$ & $g$ & sample size & $L\left(p_{0}\right)$ & $c$ & $k$ & $g$ & sample size & $L\left(p_{0}\right)$ \\
\hline \multirow{4}{*}{0.25} & 2 & 1 & 3 & 4 & 40 & 0.9656 & 3 & 1 & 2 & 20 & 0.9926 \\
\hline & 4 & 1 & 2 & 2 & 20 & 0.9875 & 2 & 1 & 1 & 10 & 0.9898 \\
\hline & 6 & $\uparrow$ & $\uparrow$ & $\uparrow$ & $\uparrow$ & 0.9974 & 0 & 1 & 1 & 10 & 0.9813 \\
\hline & 8 & $\uparrow$ & $\uparrow$ & $\uparrow$ & $\uparrow$ & 0.9992 & $\uparrow$ & $\uparrow$ & $\uparrow$ & $\uparrow$ & 0.9937 \\
\hline \multirow{4}{*}{0.10} & 2 & 2 & 13 & 14 & 140 & 0.9912 & 4 & 2 & 2 & 20 & 0.9594 \\
\hline & 4 & 0 & 2 & 3 & 30 & 0.9631 & 2 & 1 & 1 & 10 & 0.9898 \\
\hline & 6 & $\uparrow$ & $\uparrow$ & $\uparrow$ & $\uparrow$ & 0.9918 & 0 & 1 & 1 & 10 & 0.9813 \\
\hline & 8 & $\uparrow$ & $\uparrow$ & $\uparrow$ & $\uparrow$ & 0.9973 & $\uparrow$ & $\uparrow$ & $\uparrow$ & $\uparrow$ & 0.9937 \\
\hline \multirow{4}{*}{0.05} & 2 & 2 & 16 & 17 & 170 & 0.9871 & 3 & 2 & 3 & 30 & 0.9792 \\
\hline & 4 & 1 & 4 & 4 & 40 & 0.9751 & 2 & 1 & 1 & 10 & 0.9898 \\
\hline & 6 & 0 & 3 & 4 & 40 & 0.9843 & 1 & 1 & 1 & 10 & 0.9813 \\
\hline & 8 & $\uparrow$ & $\uparrow$ & $\uparrow$ & $\uparrow$ & 0.9947 & $\uparrow$ & $\uparrow$ & $\uparrow$ & $\uparrow$ & 0.9937 \\
\hline \multirow{4}{*}{0.01} & 2 & 2 & 22 & 23 & 230 & 0.9770 & 3 & 3 & 4 & 40 & 0.9607 \\
\hline & 4 & 1 & 6 & 6 & 60 & 0.9629 & 1 & 1 & 2 & 20 & 0.9936 \\
\hline & 6 & 0 & 3 & 4 & 40 & 0.9843 & 1 & 1 & 1 & 10 & 0.9813 \\
\hline & 8 & $\uparrow$ & $\uparrow$ & $\uparrow$ & $\uparrow$ & 0.9947 & $\uparrow$ & $\uparrow$ & $\uparrow$ & $\uparrow$ & 0.9937 \\
\hline
\end{tabular}

note) The upward arrow $(\uparrow)$ indicates that the same value of the parameter as in the upward cell applies to the corresponding cell.

Table 5: Proposed plan for Weibull distribution with $m=3$ when $r=5$

\begin{tabular}{|c|c|c|c|c|c|c|c|c|c|c|c|c|}
\hline \multirow{2}{*}{$\beta$} & \multirow{2}{*}{$\begin{array}{c}\mu / \mu_{0} \\
=r_{2}\end{array}$} & \multicolumn{5}{|c|}{$a=0.5$} & & \multicolumn{5}{|c|}{$a=1.0$} \\
\hline & & $c$ & $k$ & $g$ & sample size & $L\left(p_{0}\right)$ & & $c$ & $k$ & $g$ & sample size & $L\left(p_{0}\right)$ \\
\hline \multirow{4}{*}{0.25} & 2 & 1 & 23 & 23 & 115 & 0.9728 & & 2 & 2 & 2 & 10 & 0.9892 \\
\hline & 4 & 0 & 4 & 4 & 20 & 0.9725 & & 1 & 1 & 1 & 5 & 0.9988 \\
\hline & 6 & $\uparrow$ & $\uparrow$ & $\uparrow$ & $\uparrow$ & 0.9918 & & $\uparrow$ & $\uparrow$ & $\uparrow$ & $\uparrow$ & 0.9999 \\
\hline & 8 & $\uparrow$ & $\uparrow$ & $\uparrow$ & $\uparrow$ & 0.9965 & & $\uparrow$ & $\uparrow$ & $\uparrow$ & $\uparrow$ & 1.0000 \\
\hline \multirow{4}{*}{0.10} & 2 & 1 & 37 & 37 & 185 & 0.9566 & & 1 & 2 & 3 & 15 & 0.9893 \\
\hline & 4 & 0 & 9 & 10 & 50 & 0.9979 & & 0 & 1 & 2 & 10 & 0.9971 \\
\hline & 6 & $\uparrow$ & $\uparrow$ & $\uparrow$ & $\uparrow$ & 0.9998 & & $\uparrow$ & $\uparrow$ & $\uparrow$ & $\uparrow$ & 0.99973 \\
\hline & 8 & $\uparrow$ & $\uparrow$ & $\uparrow$ & $\uparrow$ & 1.0000 & & $\uparrow$ & $\uparrow$ & $\uparrow$ & $\uparrow$ & 1.0000 \\
\hline \multirow{4}{*}{0.05} & 2 & 1 & 75 & 76 & 380 & 0.9961 & & 1 & 3 & 4 & 20 & 0.9795 \\
\hline & 4 & 0 & 7 & 7 & 35 & 0.9525 & & 1 & 2 & 2 & 10 & 0.9976 \\
\hline & 6 & $\uparrow$ & $\uparrow$ & $\uparrow$ & $\uparrow$ & 0.9857 & & $\uparrow$ & $\uparrow$ & $\uparrow$ & $\uparrow$ & 0.9998 \\
\hline & 8 & $\uparrow$ & $\uparrow$ & $\uparrow$ & $\uparrow$ & 0.9939 & & $\uparrow$ & $\uparrow$ & $\uparrow$ & $\uparrow$ & 1.0000 \\
\hline \multirow{4}{*}{0.01} & 2 & 1 & 105 & 106 & 530 & 0.9927 & 1 & 4 & 5 & 25 & 0.9672 & \\
\hline & 4 & 0 & 15 & 16 & 80 & 0.9946 & 0 & 2 & 3 & 15 & 0.9915 & \\
\hline & 6 & $\uparrow$ & $\uparrow$ & $\uparrow$ & $\uparrow$ & 0.9995 & & $\uparrow$ & $\uparrow$ & $\uparrow$ & $\uparrow$ & 0.9992 \\
\hline & 8 & $\uparrow$ & $\uparrow$ & $\uparrow$ & $\uparrow$ & 0.9999 & & $\uparrow$ & $\uparrow$ & $\uparrow$ & $\uparrow$ & 0.9999 \\
\hline
\end{tabular}

note) The upward arrow $(\uparrow)$ indicates that the same value of the parameter as in the upward cell applies to the corresponding cell.

termination times are considered ( $a=0.5$ and $a=1.0$ ). The sample size and the lot acceptance probability at the producer's risk were also included in this table.

Table 4 is the similar table under Weibull distribution with $m=2$ for the proposed plan with $r=10$. Other table setting is same as in Table 3. Table 5 and Table 6 are under Weibull distribution with $m=3$ for $r=5$ and $r=10$, respectively.

From these tables it is clear that (1) as mean ratio is increased for fixed values of $r, m$ and $a$, we noted the decreasing trends in parameters, probability of acceptance and the ASN. The ASN is decreased when the experiment time is increased from 0.5 to 1.0 keeping other values at same (2) for the same values $m$ and $a$, as $r$ is increased from 5 to 10 , the ASN is as well as design parameter decreased. Finally, for the same values of $a$ and $r$, as the shape parameter is increased, we observed 
Table 6: Proposed plan for Weibull distribution with $m=3$ when $r=10$

\begin{tabular}{|c|c|c|c|c|c|c|c|c|c|c|c|}
\hline \multirow{2}{*}{$\beta$} & \multirow{2}{*}{$\begin{array}{l}\mu / \mu_{0} \\
=r_{2}\end{array}$} & \multicolumn{5}{|c|}{$a=0.5$} & \multicolumn{5}{|c|}{$a=1.0$} \\
\hline & & $c$ & $k$ & $g$ & sample size & $L\left(p_{0}\right)$ & $c$ & $k$ & $g$ & sample size & $L\left(p_{0}\right)$ \\
\hline \multirow{4}{*}{0.25} & 2 & 1 & 6 & 6 & 60 & 0.9692 & 2 & 1 & 1 & 10 & 0.9530 \\
\hline & 4 & 0 & 2 & 2 & 20 & 0.9726 & 1 & 1 & 1 & 10 & 0.9948 \\
\hline & 6 & $\uparrow$ & $\uparrow$ & $\uparrow$ & $\uparrow$ & 0.9918 & $\uparrow$ & $\uparrow$ & $\uparrow$ & $\uparrow$ & 0.9995 \\
\hline & 8 & $\uparrow$ & $\uparrow$ & $\uparrow$ & $\uparrow$ & 0.9965 & $\uparrow$ & $\uparrow$ & $\uparrow$ & $\uparrow$ & 0.9999 \\
\hline \multirow{4}{*}{0.10} & 2 & 1 & 17 & 18 & 180 & 0.9961 & 2 & 1 & 1 & 10 & 0.9530 \\
\hline & 4 & 0 & 3 & 3 & 30 & 0.9591 & 1 & 1 & 1 & 10 & 0.9948 \\
\hline & 6 & $\uparrow$ & $\uparrow$ & $\uparrow$ & $\uparrow$ & 0.9877 & $\uparrow$ & $\uparrow$ & $\uparrow$ & $\uparrow$ & 0.9995 \\
\hline & 8 & $\uparrow$ & $\uparrow$ & $\uparrow$ & $\uparrow$ & 0.9948 & $\uparrow$ & $\uparrow$ & $\uparrow$ & $\uparrow$ & 0.9998 \\
\hline \multirow{4}{*}{0.05} & 2 & 1 & 20 & 21 & 210 & 0.9947 & 2 & 1 & 1 & 10 & 0.9530 \\
\hline & 4 & 0 & 5 & 6 & 60 & 0.9972 & 1 & 1 & 1 & 10 & 0.9948 \\
\hline & 6 & $\uparrow$ & $\uparrow$ & $\uparrow$ & $\uparrow$ & 0.9997 & $\uparrow$ & $\uparrow$ & $\uparrow$ & $\uparrow$ & 0.9995 \\
\hline & 8 & $\uparrow$ & $\uparrow$ & $\uparrow$ & $\uparrow$ & 1.0000 & $\uparrow$ & $\uparrow$ & $\uparrow$ & $\uparrow$ & 0.9999 \\
\hline \multirow{4}{*}{0.01} & 2 & 1 & 29 & 30 & 300 & 0.9893 & 3 & 3 & 3 & 30 & 0.9784 \\
\hline & 4 & 0 & 8 & 9 & 90 & 0.9936 & 1 & 1 & 1 & 10 & 0.9948 \\
\hline & 6 & $\uparrow$ & $\uparrow$ & $\uparrow$ & $\uparrow$ & 0.9994 & $\uparrow$ & $\uparrow$ & $\uparrow$ & $\uparrow$ & 0.9995 \\
\hline & 8 & $\uparrow$ & $\uparrow$ & $\uparrow$ & $\uparrow$ & 0.9999 & $\uparrow$ & $\uparrow$ & $\uparrow$ & $\uparrow$ & 0.9999 \\
\hline
\end{tabular}

note) The upward arrow $(\uparrow)$ indicates that the same value of the parameter as in the upward cell applies

$$
\text { to the corresponding cell. }
$$

Table 7: Sample sizes in two group sampling plans for Weibull distribution with $m=2(a=0.5)$

\begin{tabular}{|c|c|c|c|c|c|}
\hline \multirow[b]{2}{*}{$\beta$} & \multirow{2}{*}{$\begin{array}{l}\mu / \mu_{0} \\
=r_{2}\end{array}$} & \multicolumn{2}{|c|}{$r=5$} & \multicolumn{2}{|c|}{$r=10$} \\
\hline & & Proposed Plan & Existing Plan $(k=g)$ & Proposed Plan & Existing Plan $(k=g)$ \\
\hline \multirow{3}{*}{0.25} & 2 & 60 & 160 & 40 & 160 \\
\hline & 4 & 20 & 30 & 20 & 20 \\
\hline & 6 & 20 & 30 & 20 & 20 \\
\hline \multirow{3}{*}{0.10} & 2 & 85 & 2655 & 140 & 260 \\
\hline & 4 & 25 & 50 & 30 & 30 \\
\hline & 6 & 25 & 50 & 30 & 30 \\
\hline \multirow{3}{*}{0.05} & 2 & 550 & 2955 & 170 & 340 \\
\hline & 4 & 30 & 65 & 40 & 40 \\
\hline & 6 & 30 & 65 & 40 & 40 \\
\hline \multirow{3}{*}{0.01} & 2 & 765 & 5315 & 230 & 520 \\
\hline & 4 & 95 & 95 & 60 & 60 \\
\hline & 6 & 40 & 95 & 40 & 60 \\
\hline
\end{tabular}

the decreasing trend in design parameters values as well as ASN but increasing trend in probability of acceptance.

Example 2. Suppose that a manufacturer wants to use the proposed group sampling plan for the inspection of incoming lots of energy saver bulbs. Multi-item tests with group size of 10 will be used. Suppose also that the life of this product follows a Weibull distribution with shape parameter of 2. It is known that the specified life of interest is 10,000 hours. The test time was specified as 5,000 hours. It is required that the consumer's risk is 10 percent if the true mean life is 10,000 and the producer's risk is 5 percent if the true mean is 40,000. As $a=0.5, \beta=0.1$ and $r_{2}=4$, it is found from Table 4 that $(g, k, c)=(3,2,0)$. So, a sample of 30 items is drawn and allocated to 3 groups. If there are no failures from at least 2 groups out of 3 , then the lot will be accepted.

In order to demonstrate the advantage of the proposed group sampling plan over the existing group sampling plan, the sample size required can be compared with each other. Table 7 compares the sample size for the proposed plan with the existing group sampling plan in Aslam and Jun (2009b) 
when the lifetime follows the Weibull distribution with shape value of 2 .

It can be seen from Table 7, the proposed plan needs smaller number of sample size than in the existing plan for the same values of shape parameter, number of testers, experiment time and the mean ratio.

\section{Concluding Remarks}

It was demonstrated that the proposed group sampling plan reduces the sample size required as compared to the existing plan by modifying the acceptance criteria. The design parameters can be determined independently of the underlying life distribution if the ARL and the LTRL are specified as unreliabilities. However, if the ARL or the LTRL should be specified as the true mean life or the mean ratio to the specified life, the underlying life distribution should be also given. Weibull distribution was considered as an example in this study, but some other various life distributions can be easily implemented similarly. There may be needed a further study of developing more efficient group sampling plans to reduce the sample size required.

\section{Acknowledgement}

The authors would like to thank the Editor and the anonymous reviewers for their valuable suggestions to improve this manuscript.

\section{References}

Aslam, M. and Jun, C.-H. (2009a). Group acceptance sampling plans for truncated life tests based on the inverse Rayleigh distribution and log-logistic distribution, Pakistan Journal of Statistics, 25, $107-119$.

Aslam, M. and Jun, C.-H. (2009b). A group acceptance sampling plan for truncated life test having Weibull distribution, Journal of Applied Statistics, 36, 1021-1027.

Balakrishnan, N., Leiva, V. and Lopez, J. (2007). Acceptance sampling plans from truncated life tests based on the generalized Birnbaum-Saunders distribution, Communications in StatisticsSimulation and Computation, 36, 643-656.

Fertig, F. W. and Mann, N. R. (1980). Life-test sampling plans for two-parameter Weibull populations, Technometrics, 22, 165-177.

Goode, H. P. and Kao, J. H. K. (1961). Sampling plans based on the Weibull distribution, Proceeding of the Seventh National Symposium on Reliability and Quality Control, Philadelphia, 24-40.

Gupta, S. S. (1962). Life test sampling plans for normal and lognormal distributions, Technometrics, 4, 151-175.

Gupta, S. S. and Groll, P. A. (1961). Gamma distribution in acceptance sampling based on life tests, Journal of the American Statistical Association, 56, 942-970.

Jun, C.-H., Balamurali, S. and Lee, S.-H. (2006). Variables sampling plans for Weibull distributed lifetimes under sudden death testing, IEEE Transactions on Reliability, 55, 53-58.

Kantam, R. R. L., Rosaiah, K. and Rao, G. S. (2001). Acceptance sampling based on life tests: Log-logistic models, Journal of Applied Statistics, 28, 121-128.

Tsai, T.-R. and Wu, S.-J. (2006). Acceptance sampling based on truncated life tests for generalized Rayleigh distribution, Journal of Applied Statistics, 33, 595-600. 